\title{
How I look at the regurgitant mitral valve-a stepwise echocardiographic assessment
}

\section{Raluca Dulgheru ${ }^{1}$, Samuel Bruls ${ }^{1}$, and Patrizio Lancellotti ${ }^{1,2 *}$}

1'Department of Cardiology and Cardiovascular Surgery, University of Liège Hospital, GIGA Cardiovascular Sciences, CHU Sart Tilman, Avenue de l'Hôpital 1, Batmiment B35, Liège, Belgium; and ${ }^{2}$ Gruppo Villa Maria Care and Research, Anthea Hospital, Camillo Rosalba, 35/37, 70124 Bari BA, Italy

Keywords mitral regurgitation $\bullet$ echocardiography $\bullet$ quantification

\section{Introduction}

Mitral regurgitation (MR) is the second most common valve disease needing intervention in the Western world, after degenerative aortic stenosis. ${ }^{1}$ Echocardiography is the key examination in the assessment of MR patients. ${ }^{2}$ Echocardiography grades MR severity, establishes its mechanism and aetiology, gives a complete morphological description of the valve, assesses the left ventricular (LV) systolic function and remodelling, the degree of left atrium (LA) dilatation, and the likelihood of pulmonary hypertension, and describes and quantifies associated valve lesions. Transoesophageal echocardiography (TOE) remains the examination of choice for the complete description of mitral valve (MV) morphology and confirmation of the MR mechanism. TOE should be performed whenever quantification by TTE is impossible. TOE is cornerstone for preprocedural assessment and procedural guiding in transcatheter valve interventions. Within the following pages the reader will find an easy to follow and userfriendly description of the main steps for the evaluation of patients with MR (Figure 1).

\section{Putting things into clinical context}

Look at the valve, but also at the LV! Evaluating the LV is important from several standpoints: (i) not to miss a severe acute MR, (ii) establish if MR is secondary, primary, or mixed, and (iii) avoid overestimation of MR severity.

\section{Acute vs. chronic MR}

Acute heart failure (HF) in a patient with a non-dilated and hyperdynamic LV should raise suspicion for acute severe primary MR. TOE should be performed whenever visualization of the $M V$ is suboptimal with TTE and the HF episode remains unexplained. Acute severe MR should not be missed as treatment changes completely. Acute primary MR can be differentiated from chronic primary MR complicated by ruptured chordae by analysis of LV geometry and size. In chronic primary $M R$, due to the longstanding volume overload of the LV, there is significant LA and LV dilatation and a rounded-shape LV apex. In acute MR, LV is usually non-dilated and hyperdynamic.

\section{Primary, secondary, or mixed MR}

$M R$ is classified into primary, secondary, and mixed MR. If any components of the MV apparatus (leaflets, chordae, annulus, papillary muscles) are structurally abnormal, then MR is considered primary. If all components are structurally normal, but MR ensues due to a distortion of the valve geometry, MR is considered secondary. When both are present, $M R$ is considered mixed. With population ageing, it is likely that the mixed form to become more prevalent. Usually, secondary MR is the result of a ventricular disease that leads to LV regional, and/or global remodelling and systolic dysfunction and, thus, to an increase in tethering forces and a decrease in closing forces acting on the MV leaflets. Hence, looking at the LV is important to pick up the hint that MR may be secondary. Wall motion abnormalities will orient towards secondary MR, or mixed MR if the leaflet's tissue is abnormal. LV dyssynchrony together with LV dilatation and dysfunction may also orient towards secondary MR. In atrial functional $M R$, the $L V$ is not dilated and MR is considered secondary. Such patients have longstanding atrial fibrillation, very dilated left and right atria, very dilated atrio-ventricular annuli, and 'atrial' functional $M R^{3}{ }^{3}$ In atrial functional MR, the annular dilatation (which pulls the leaflets apart) and the increase in LA pressure (which pushes the leaflets apart) are the main promoters of MV incompetence. Patients with hypertrophic obstructive cardiomyopathy (HOCM) may also have secondary/mixed MR. MR in HOCM is highly dynamic, with a regurgitant jet (Reg))-oriented posteriorly and related to the destabilization of the coaptation surface (CS) by leaflet tethering towards the interventricular septum in midsystole. However, in some patients with HOCM, there are also some structural abnormalities of the valve leaflets (long leaflets) and of the subvalvular apparatus (abnormally implanted papillary muscles and aberrant mitral chordae), leading to MR. In this case, MR should be probably labelled as mixed.

* Corresponding author. E-mail: plancellotti@chuliege.be

Published on behalf of the European Society of Cardiology. All rights reserved. (c) The Author(s) 2020. For permissions, please email: journals.permissions@oup.com. 


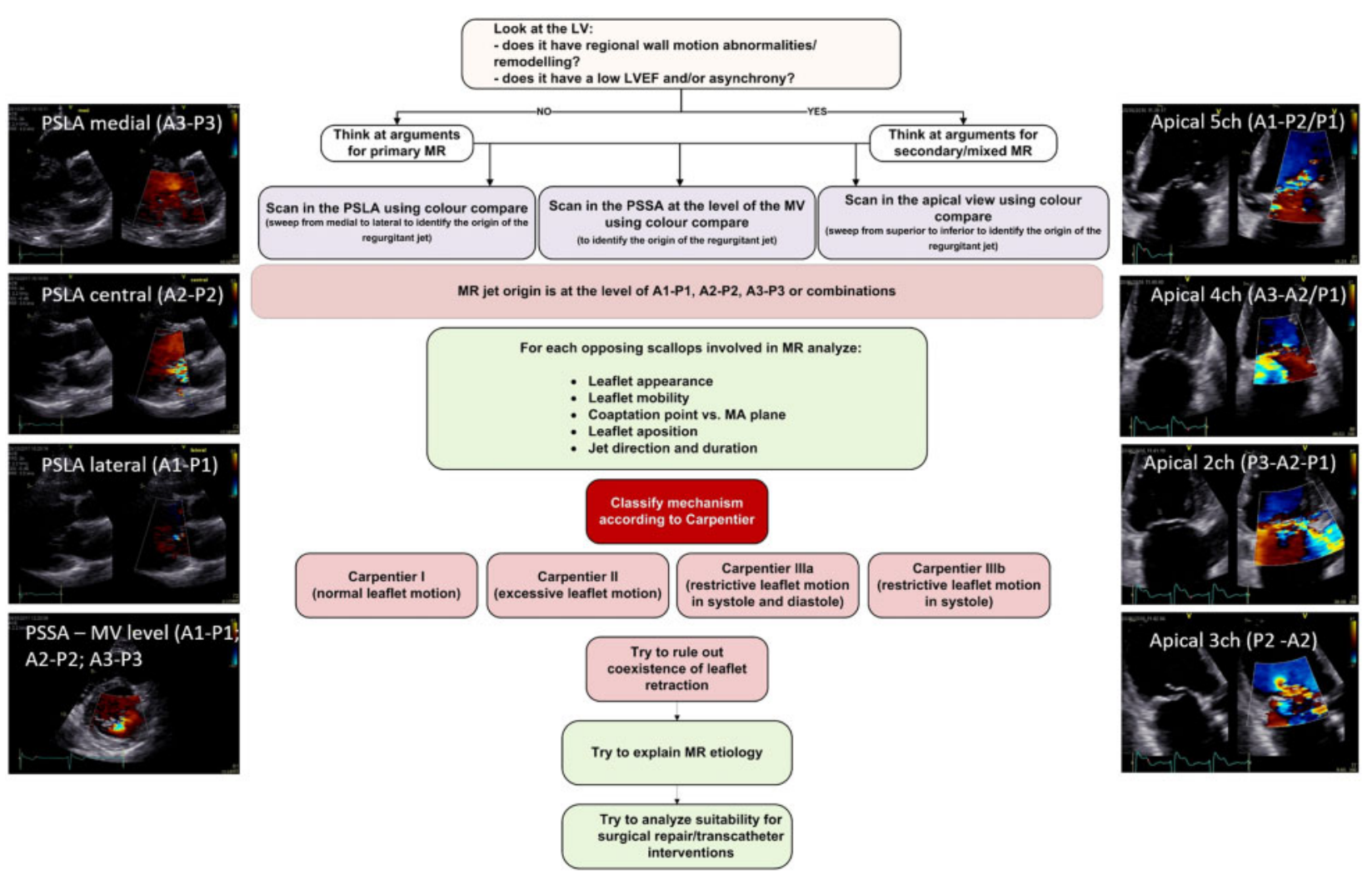

Figure I Flow chart depicting the main steps in MR evaluation by TTE. The images depict how the systematic scanning of the MV using TTE, 2D and Colour Flow Doppler and colour compare mode is performed with TTE. PSLA, parasternal long axis; PSSA, parasternal short axis; 4ch, four chambers; 2 ch, two chambers; 3 ch, three chambers.

\section{Severe vs. non-severe MR}

Each attempt to quantify MR severity has to be done together with a complete examination of the LV and the LA. Primary MR is very likely non-severe if LA and LV are not dilated and acute MR has been excluded. MR quantification should be reattempted in such a case.

\section{Severity quantification in practice}

Quantification of MR severity is important as intervention is considered only for patients having severe $M R^{2}{ }^{2}$

Since MR occurs as a result of abnormal coaptation between the two MV leaflets, rigorous characterization of the CS and detection of the scallops involved in MR (segmental analysis) will help to quantify MR severity accurately and understand its aetiology and mechanism.

The first step is to identify the weakest point of coaptation (WPC) along the CS of the leaflets, thus the origin of the RegJ. This means that coaptation line has to be scanned from multiple acoustic windows systematically both in TTE and TOE, ideally using colour compare mode (the side-by-side display of 2D image with/without colour). At the WPC, one should be able to identify with colour Doppler the convergence zone and the vena contracta of the Rrg] (Figure 1). After this step, one should be able to understand the origin of the Reg/jets with respect to the MV scallops: A1-P1, A2-P2, A3P3, or combinations (in case of multiple jets or jets extended over several scallops). This segmental analysis of the coaptation line is important because several mechanisms of MR may coexist.

Identifying the WPC (i.e. the origin of the Reg)) is crucial for several reasons: (i) from this cut-plane starts the identification of the mechanism of the MR by analysing leaflet motion, leaflet apposition, leaflet morphology, and (ii) will improve MR quantification.

Once the WPC has been identified, quantification of severity using semi-quantitative or quantitative methods can be attempted. All quantification methods have been described in other publications, and will not be reviewed here. ${ }^{4}$ However, some simple principles regarding the MR quantification will be reminded. Identification of a ruptured chordae is usually indicative of a severe primary MR. In such cases, quantification of MR with the PISA method is not mandatory since MR is obviously severe or very severe. Moreover, in such cases, the PISA method may not be very accurate. Identification of a large flow convergence hemisphere, with colour flow Doppler, without baseline shift, should alert of severe MR. Likewise, identification of a large eccentric regurgitant jet tapering and swirling around the walls of the LA. On the other hand, a dominant A wave of the mitral inflow, in the absence of mitral stenosis, rules out severe MR. Nonholosystolic MR is rarely severe. 


\section{Understanding mechanism: basic steps}

Understanding of the mechanism of MR comes from the attentive observation of leaflet movement at the WPC, but not only. Aetiology and mechanism of MR are very closely linked. Hence, identification of MR aetiology will also help in understanding its mechanism and viceversa.

LV evaluation should be performed prior to the attempt of visualization of valve morphology, as this will orient towards a secondary, primary or mixed MR type.

Classically, MR mechanism is described according to the Carpentier classification. This classification is based on the pattern of $M V$ leaflet motion. However, it does not consider leaflet retraction (decrease in MV leaflet surface), which can contribute to a significant decrease of the coaptation reserve and hence, to MR. It has been shown that $M V$ leaflets can increase their surface in response to leaflet tethering, so that coaptation to be preserved. ${ }^{5}$ The inverse situation, when there is leaflet fibrosis and calcification-both retractile processes - coaptation reserve may be reduced and MR aggravated. Identifying leaflet retraction is important because it may impact on the likelihood of MV repair procedures. Usually, once there is lack leaflet of tissue, the valve is very likely difficult, if not impossible, to repair.

For the next step in the analysis, for each opposing scallops involved in the $M R$, the coaptation point with regard to the mitral annulus (MA) plane is analysed from a long axis image plane cutting through the origin of the Reg). The position of each leaflet tip as compared to its ipsilateral MA is analysed. Coaptation point apically displaced towards the LV suggests systolic leaflet restrictive motion (Carpentier IIlb or IIla). If leaflets are not moving freely in diastole and chordae seem short and thickened, then a Carpentier Illa mechanism should be described. Coaptation point above the MA plane, into the LA, is highly suggestive of excessive leaflet motion (Carpentier II). Second step is to look at the way the tips of the mitral leaflets face each other during systole, i.e. leaflet apposition. The way leaflet's tips align with each other in systole will give the Regj direction. Symmetrical leaflet apposition (edge-to-edge) can be indicative of a mechanism that pulls the leaflet apart symmetrically and this is usually related to MA dilatation, Carpentier I, or to symmetric bileaflet systolic tethering, Carpentier IIlb. Rarely, this may be found in bileaflet prolapse, hence Carpentier II. Usually, MR jet is directed centrally in the LA in this case. Asymmetrical leaflet apposition (body-to-edge) may be encountered in asymmetric leaflet restrictive motion (Carpentier Illa or IIlb) or in MV prolapse (Carpentier II). Asymmetrical leaflet apposition leads to eccentric MR jets. To note, in complex forms of MR with multiple MR jets, several different mechanisms may be identified. Hence, the importance of segmental analysis.

Likewise, MR jet direction helps in understanding its mechanism. In $M V$ prolapse or flail leaflet, the MR jet is directed away from the flail/ prolapsing scallop, with some exceptions: bileaflet symmetric prolapse (jet may be central) or commissural prolapse (variable direction but very eccentric jets). In MR due to restrictive systolic motion, jet is directed behind the restricted scallop. When tethering acts symmetrically on the MV leaflets, Regl may expand somehow more centrally in the LA. The type of leaflet apposition will give jet direction. In atrial functional MR, Reg is usually also central. Functional MR in HOCM has a posteriorly oriented MR jet.

Conflict of interest: none declared.

\section{References}

1. lung B, Vahanian A. Epidemiology of acquired valvular heart disease. Can J Cardiol 2014;30:962-70.

2. Baumgartner H, Falk V, Bax JJ, De Bonis M, Hamm C, Holm PJ et al.; ESC Scientific Document Group. 2017 ESC/EACTS Guidelines for the management of valvular heart disease. Eur Heart J 2017;38:2739-86.

3. Silbiger JJ. Mechanistic insights into atrial functional mitral regurgitation: far more complicated than just left atrial remodeling. Echocardiography 2019;36:164-9.

4. Lancellotti P, Tribouilloy C, Hagendorff A, Popescu BA, Edvardsen T, Pierard LA et al.; On behalf of the Scientific Document Committee of the European Association of Cardiovascular Imaging: Thor Edvardsen, Oliver Bruder, Bernard Cosyns, Erwan Donal, Raluca Dulgheru, Maurizio Galderisi, Patrizio Lancellotti, Denisa Muraru, Koen Nieman, Rosa S. Recommendations for the echocardiographic assessment of native valvular regurgitation: an executive summary from the European Association of Cardiovascular Imaging. Eur Heart J Cardiovasc Imaging 2013;14:611-44.

5. Chaput M, Handschumacher MD, Tournoux F, Hua L, Guerrero JL, Vlahakes GJ et al. Mitral leaflet adaptation to ventricular remodeling occurrence and adequacy in patients with functional mitral regurgitation. Circulation 2008;118:845-52. 